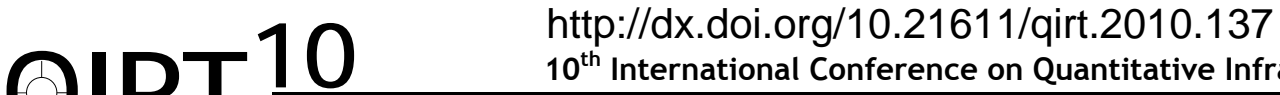 \\ $10^{\text {th }}$ International Conference on Quantitative InfraRed Thermography \\ July 27-30, 2010, Québec (Canada)
}

\section{Automated defect detection in large-scale bonded parts by active thermography}

\author{
by C. Thiemann*, M. F. Zaeh*, C. Srajbr** and S. Boehm ${ }^{\star \star}$ \\ * Institute for Machine Tools and Manufacturing Technology (iwb), Technische Universitaet Muenchen, Germany \\ ** Department of adhesive bonding, Institute for Joining and Welding (ifs), Technische Universitaet Braunschweig
}

\begin{abstract}
Currently, no feasible testing methods are available which allow a cost-effective and in-line non-destructive testing of adhesive bonds. However, thermography has a great potential for the detection of defects in a fast and reliable way. Limitations of the resolution of commercially available infrared cameras usually require a repositioning of the setup to inspect the surface of large parts. This paper presents a new concept for an automated testing method for the thermographic inspection of large-scale bonded parts, which is based on scanning strategies using infrared mirrors. The concept was evaluated by an industrial reference application.
\end{abstract}

\section{Introduction}

Quality evaluation of adhesive bonds can be carried out either by destructive or non-destructive testing procedures. However, destructive testing of random samples, which is usually state of the art in industrial mass production of bonded parts, is cost-intensive and requires much effort. Thus, the enhancement of non-destructive testing methods with respect to a reliable detection of all quality-related defects, short testing periods and a high automation level is of great economical and technical importance for ensuring the operational capability of adhesive bonded parts.

Active thermography is a suitable non-destructive testing procedure for the adhesive technology. In recent years, a couple of research institutes provided evidence that this method is applicable for the inspection of adhesive bonds [1-5]. However, an implementation in industrial mass production is still impeded due to deficiencies such as unavailable concepts for testing large and complex components as well as for reduction of testing time. The objective of a recent research project, which was funded by the Bayerische Forschungsstiftung, was the development of a testing system based on thermography that is capable of analyzing the quality of bonded products in mass production within an acceptable cycle time. This implies a reliable detection of defects in industrial environments as well as the application of cost-efficient components, which will also favor the use of non-destructive testing systems in the small business sector. Based on industrial reference applications different concepts for the automation of the thermographic testing process have been developed and successfully applied.

In contrast to thermographic inspections of welded joints, which have already successfully been introduced into industrial environment [6,7], adhesive bonds behave differently. Figure 1 shows a typical composition of an adhesive bond, which consists of up to three different kinds of material that build two interfaces.

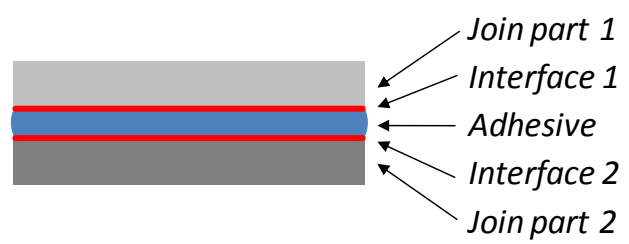

Fig. 1. Composition of adhesive bonds

As a consequence, thermographic inspection faces a complex thermal behavior that influences the detectability of possible defects which can occur in both of the bounding surfaces as well as in the adhesive itself. Additionally, the attainable contrast between defect and sound area is smaller than in welded joints, because the heat transfer characteristic of the composition is only little affected by defects. The adhesive layer, which builds a plastic in cured condition, has usually a slow response time for a thermal stimulation, i.e. the maximum attainable contrast of defects can be observed later as it would be in welded joints for example. Hence, the testing time using common inspection procedures and technologies is physically limited and can exceed, depending on the material composition and the thicknesses of the layers, up to several minutes for a single field of view. 


\section{Testing methods for large-scale parts}

\subsection{Automated thermographic testing procedures}

State-of-the-art infrared cameras have a detector array resolution of up to one Megapixel. The size of the attainable field of view on the object depends on the necessary resolution for the smallest defect that needs to be detected. For example, if the test object has dimensions of $1000 \mathrm{~mm} \times 1000 \mathrm{~mm}$ and the smallest relevant defect has a size of $1 \mathrm{~mm}^{2}$, at least nine single section measurements are necessary to obtain a pixel-to-defect-ratio of about 3.3. This value is regarded as a minimum value that allows a separation of the defect from any occurring noise. Therefore, in this paper test objects are considered as large-scale objects if more than a single measurement is required to obtain a reasonable defect resolution.

In contrast to a manual test configuration, where the infrared camera and the object are fixed and a manual reconfiguration is necessary, an automated test setup requires an automatic movement of the field of view to fulfill the aforementioned constraint. There are three basic principles that allow the motion of a camera's field of view (figure 2). The first concept is based on the intermittent or continuous movement of the camera along the object's surface [8]. In the second method, the object itself is moved in front of a stationary camera which results in the same relative movement as in the first concept $[9,10]$. A third method that has been developed at the iwb uses a scanning mirror to observe the surface of a fixed object. The thermal stimulation for each method, which is not shown in the graphic, can be realized either by a full-sized stimulation or by a moving heat source.

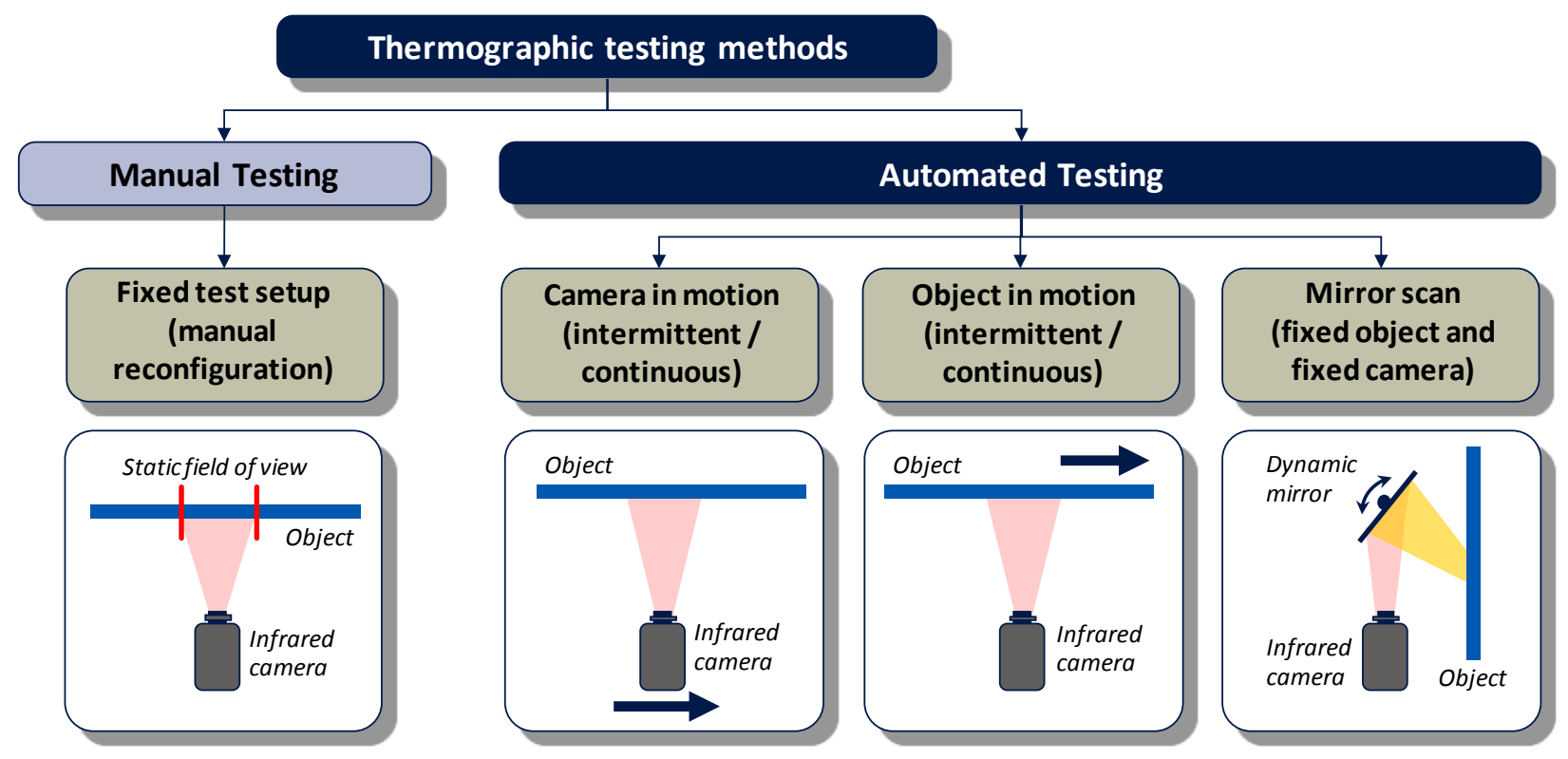

Fig. 2. Classification of automated and manual thermographic testing methods

Apart from the aforementioned solutions it is also possible to use infrared cameras with higher resolutions or more than a single camera to observe all relevant regions of the object. However, the potential for the development of infrared cameras with larger detector arrays seems to be limited in the next years, and the use of several cameras is for most applications too expensive. The following chapter presents the functional principle of the concept that is based on the use of dynamic mirrors.

\subsection{Thermographic testing by mirror scan}

Figure 3 shows the basis configuration for thermographic mirror scanning of an object after its relevant areas of interest have been thermally stimulated. A technical solution for the thermal stimulation of large areas will be presented later on for a reference application. Thereafter, the field of view of the infrared camera can be positioned on the object's surface by an adjustable mirror for infrared radiation. In a basic implementation the field of view moves sequentially across the surface after completion of the measurement of each section. However, if thermal response behavior of the adhesive bond is slow, as it can be observed in thick adhesive layers, the total testing time can cumulate up to half an hour, depending on the number of sections to be tested. Such a long testing time is not acceptable for in-line non-destructive testing in industrial mass production. 


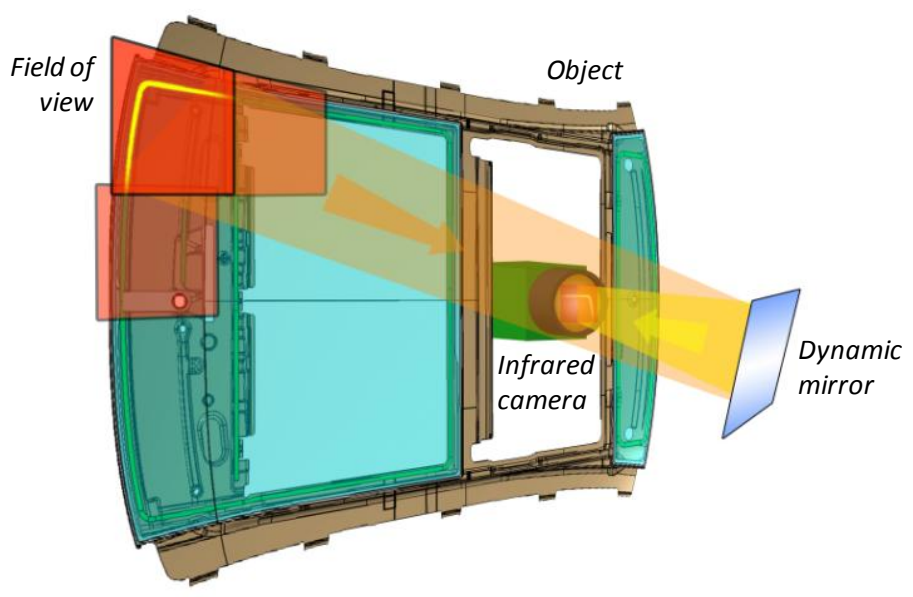

Fig. 3. Scanning thermography using a dynamic mirror

Therefore, a new mirror scan concept, which has been developed at the iwb, uses the parallelization of the measurements for different sections, as it is explained in figure 4. The large area of the test object is first separated into smaller sections which allow a suitable resolution of the infrared camera. After a pulsed thermal stimulation a scanning mechanism starts to take images in multiple measuring cycles lasting each about $0.5 \mathrm{~s}$, depending on the velocity of the kinematics and the integration time of the camera. By this, discrete measuring values of the heating (or cooling) curves are recorded for each section of the object's surface. As a consequence, the frame rate of the infrared camera for a certain section is reduced by a factor that depends on the number of sections to be scanned. For example, if the frame rate of the camera is $50 \mathrm{~Hz}$ and ten sections need to be tested, the actual frame rate for a single section results to $5 \mathrm{~Hz}$. However, adhesive bonds have a slow thermal response time and no volatile signal response of defects occurs, so that the measurement is still precise enough to allow an interpolating reconstruction of the real heating (or cooling) curve.

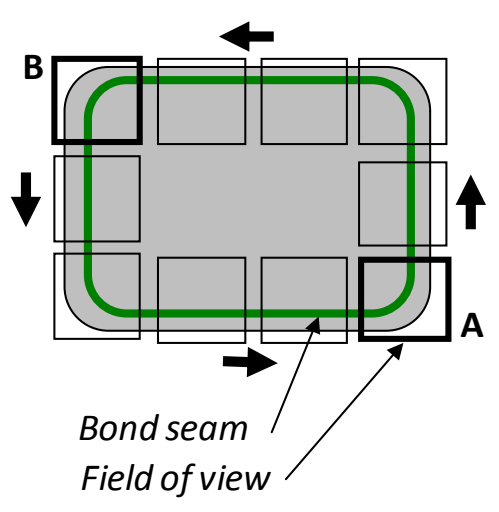

- Thermal excitation

- Thermal response

- Measuring points

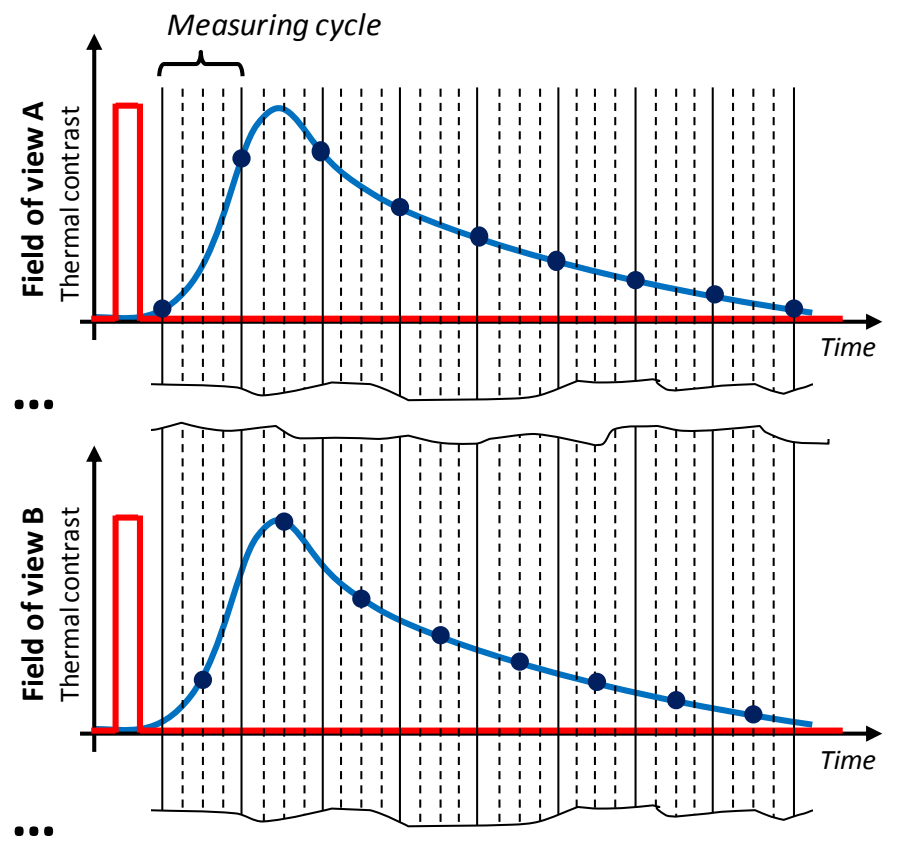

Fig. 4. Functional principle of scanning method with multiple measuring cycles

The content of the raw thermal sequence that is recorded by the infrared camera is explained in figure 5 . It contains packages of thermal section images that were gained during the circumferential measurement cycles. In a first step the images that belong to a certain field of view respectively to a certain object's section need to be separated, so that 
autonomous thermal sequences can be extracted. Hereafter follow state-of-the-art computation algorithms such as the Fourier transform to generate amplitude and phase images.

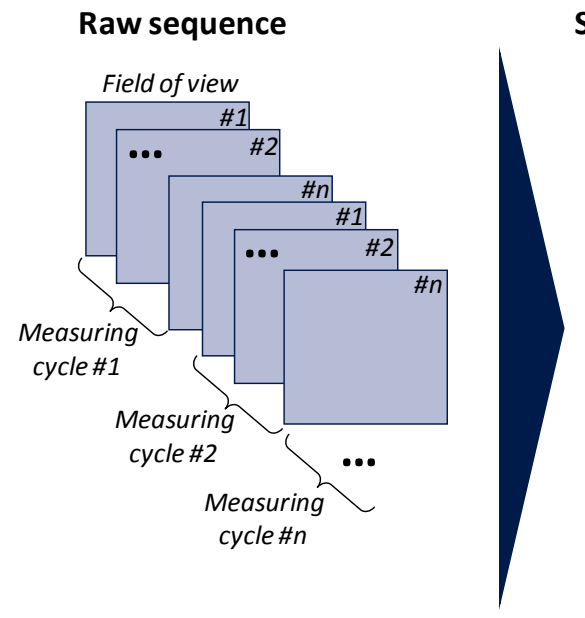

Sequence extraction
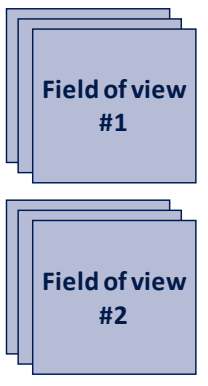

$\bullet \bullet$

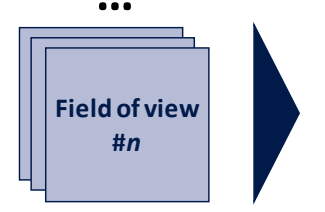

Fourier transform
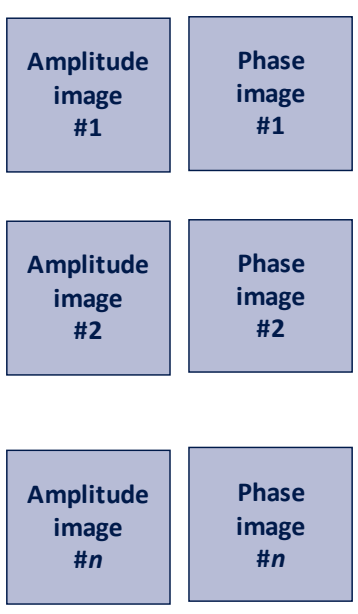

Fig. 5. Sequence reconstruction for scanning mirror method and sequence processing by Fourier transform

\section{Reference application: bonded window module}

\subsection{Application and testing requirements}

The reference application that was evaluated using the mirror scan method is a bonded glass element module used in the automobile industry. The module consists of a steel frame structure, one movable and two bonded glass elements plus numerous mounted parts. The bonded joint that is to be analyzed in this assembly joins the steel frame with the glass elements and consists of highly elastic polyurethane (Figure 6).

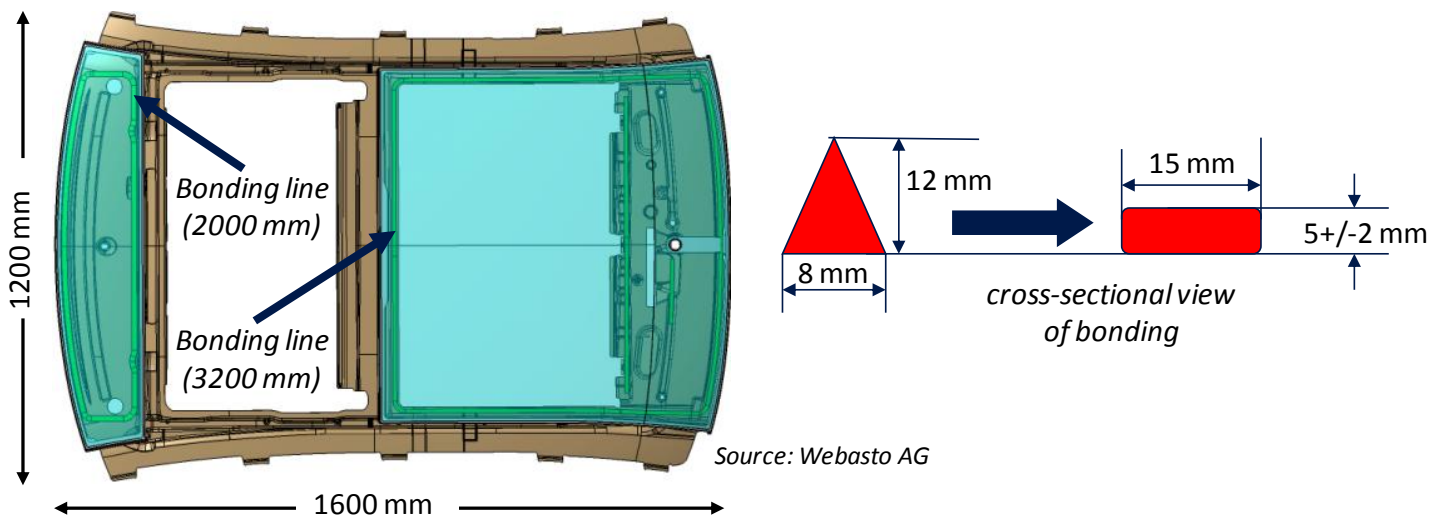

Fig. 6. Reference application "bonded window module" (automotive industry)

In order to ensure stability and leak tightness of the bonded joint, a quality inspection must detect all relevant defects in a measurement cycle time of less than three minutes. Currently, a high amount of manual effort ensures the quality of the glass modules. An automated testing process makes high demands on robustness and reliability, because the structure of the parts is complex. Furthermore, the properties of the adhesive bond vary within a single module or between different modules, each depending on the part tolerances. For example, the thickness of the adhesive bond line depends on the amount of distortion of the steel frame as well as of the glass elements.

The complexity of the steel frame structure and the numerous mounted parts which are present at the optimum process testing time do not allow a testing from the reverse side of the glass element module, because possible defects would be suppressed by existing thermal structures. Therefore, it is required to apply both thermal excitation and 
measurement equipment on the glass side. Theoretically, there are four methods of excitation: Optical or thermal radiation, ultrasound excitation or excitation by induction. Due to a low heat conductivity of the adhesive and the glass elements which have a thickness of about $5 \mathrm{~mm}$ each, a high amount of energy is required to visualize defects on the surface of the glass element. An optical excitation is not useable due to the highly reflective properties of the glass elements. Former research activities using ultrasound excitation for polyurethane bonds showed that deep defects cannot be easily detected, because the adhesive has a high degree of attenuation. A large amount of energy can be best introduced into the adhesive bond by induction heating of the steel frame, which had been proved on samples at an earlier stage of the project. During the testing process, a maximum temperature of about $100^{\circ} \mathrm{C}$ is allowed to prevent the adhesive bond from any damage.

In order to conduct the thermographic testing with realistic terms, defective samples with similar characteristics to the real products have been prepared in cooperation with the industrial partner of this project. All defects, which might be relevant in the real production process, have been realized in different sizes and locations in the adhesive bond. Preliminary tests with different methods of defect realization have been carried out and the samples have been validated with destructive testing. Quality relevant defects are kissing bonds, which are characterized by a close contact between adhesive and adherend, but have no or little adhesion, as well as interfacial unbonding, air entrapment, and also porosity and interception of the bond seam.

\subsection{Thermographic test setup}

The primarily components of the thermographic test setup developed at the iwb Application Centre Augsburg are shown in figure 7. Heating of the steel structure in the region of the adhesive is carried out by a linear inductor, which is moved with a velocity of $100 \mathrm{~mm} / \mathrm{s}$ at a distance of $2 \mathrm{~mm}$ perpendicular to the glass surface by an articulated robot. A maximum temperature at the rear side of the steel frame of about $50^{\circ} \mathrm{C}$ was measured. The thermal flow that is caused by eddy currents in the steel frame propagates through the adhesive and the glass elements, so that a transmission measurement setup is present. The infrared camera made by Thermosensorik $\mathrm{GmbH}$ has a resolution of 256 pixels $\times 256$ pixels and a maximum frame rate of $50 \mathrm{~Hz}$; the minimum temperature sensitivity is about $90 \mathrm{mK}$. The field of view, which has been observed during a single thermographic measurement cycle, has the dimensions of $250 \mathrm{~mm} \times 250 \mathrm{~mm}$. A full circulation of the inductive element as a first step prior to the scanning measurement takes about $25 \mathrm{~s}$ which induces enough heat into the adhesive bond to gain a sufficient signal-to-noise ratio. The time for thermal stimulation of the adhesive bond is low compared to the thermal response time of any hidden defects. After the heating process has been accomplished, the mirror starts to scan the surface in multiple measuring cycles. The scanning kinematics allows a highly accurate repositioning of the camera's field of view within $0.4 \mathrm{~s}$. During a total test period of $120 \mathrm{~s}$ in each of the 17 scanned sections of the object 18 images could be recorded and evaluated with the aforementioned reconstruction algorithms. The maximum contrast of the deepest defects was attained $120 \mathrm{~s}$ after thermal stimulation.
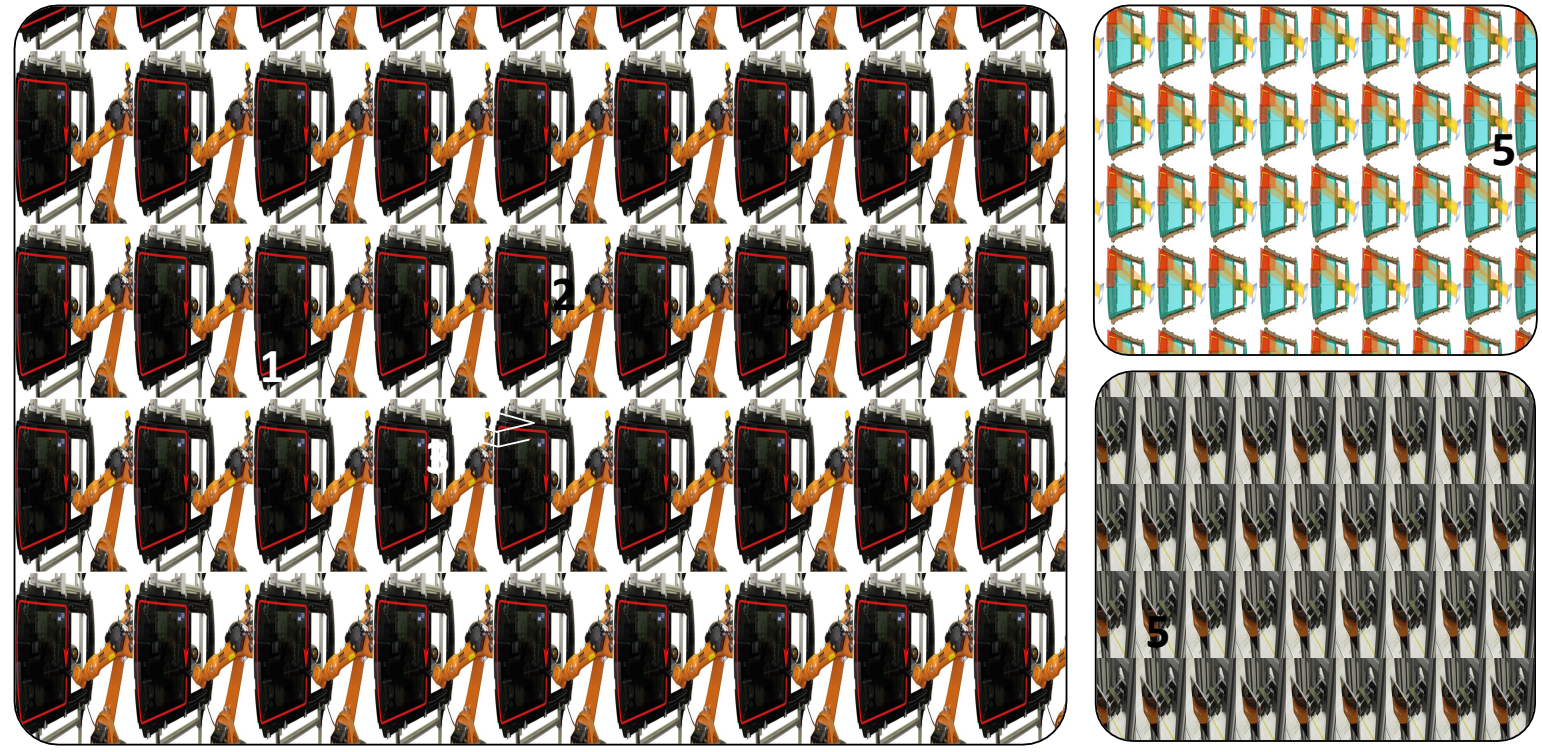

Fig. 7. Test setup for thermographic mirror scan; left: thermal excitation by inductive element; right: scanning method and scanning mechanism (1: bonded window module; 2: infrared camera; 3: inductive element; 4: kinematics; 5: mirror) 


\subsection{Results of thermographic testing}

Figure 8 shows selected results of the thermographic analysis of a defective glass element module using the aforementioned scanning method.
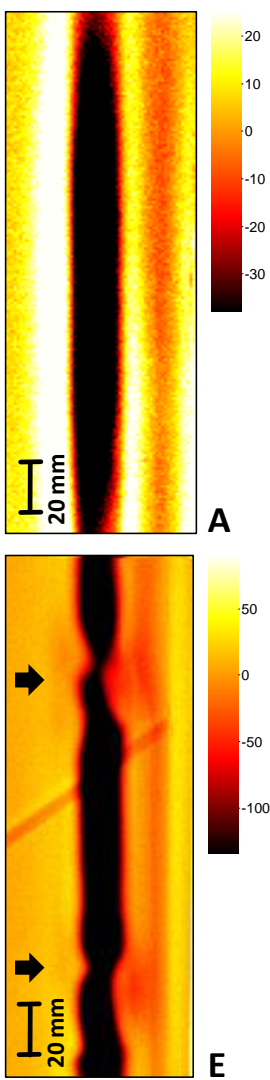

E
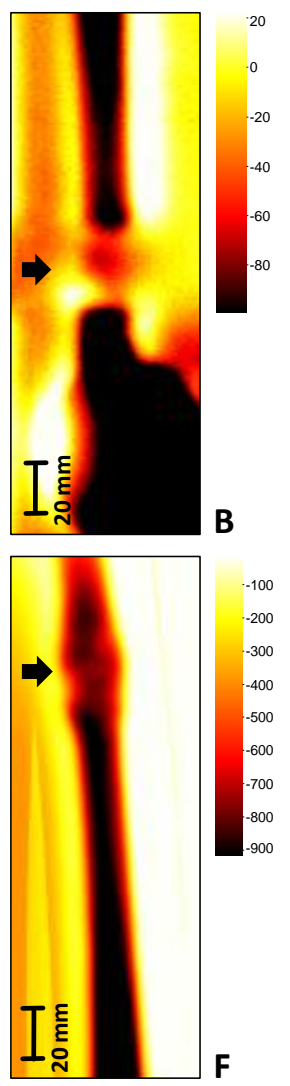
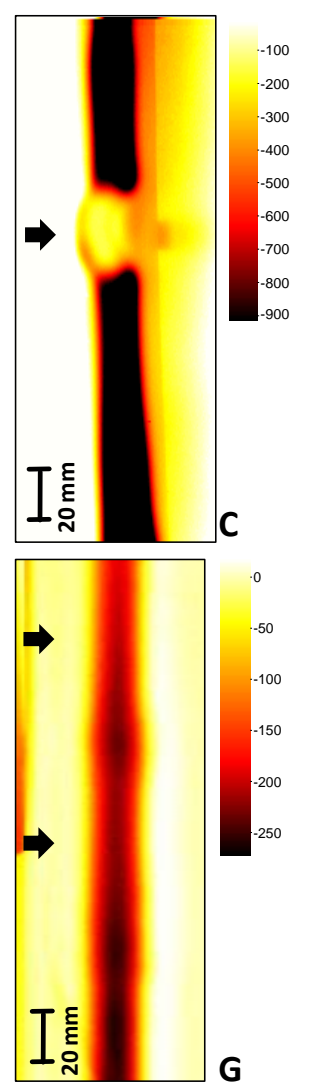

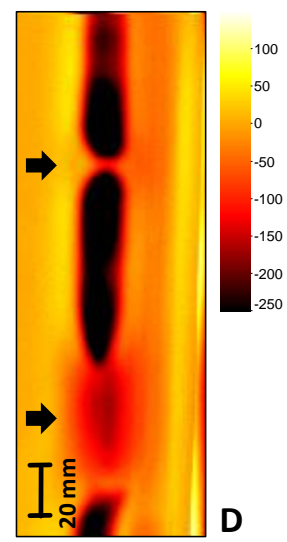

Measurement parameters:

- inductive heating of steel frame in motion

( $v=100 \mathrm{~mm} / \mathrm{s}$ )

- $\mathrm{T}_{\max }=50{ }^{\circ} \mathrm{C}$ )

- $\mathrm{T}=120 \mathrm{~s}$

- Fourier transform (amplitude-phase-images)

A sound area of adhesive bond

B interfacial unbonding ( $30 \mathrm{~mm}$; glass side)

C interfacial unbonding ( $30 \mathrm{~mm}$; frame side)

D interruption $(5 \mathrm{~mm} / 10 \mathrm{~mm}$ )

E necking $(5 \mathrm{~mm} / 10 \mathrm{~mm})$

F porosity $(2 \mathrm{~mm})$

G kissing bond ( $30 \mathrm{~mm} / 20 \mathrm{~mm}$; glass side)

Fig. 8. Thermographic results of selected defects

Figure $8(A)$ illustrates a reference region of the part with an ideal adhesive bonding line, which is clearly distinguishable from adjacent part structures. Figures 8(B) to 8(E) show the thermographic results of interfacial unbonding on glass side and steel side as well as neckings and interruptions, which are also all clearly with high contrast detectable. This is an important requirement for further steps that aim for automatization of the testing process. In the lower half of figure 8(B) an overlapping of the circular adhesive bond line can be identified as a large dark region. In the upper region of figure 8(F) porosity respectively the effect of small polystyrene balls used for defect simulation can be seen. These defects can also be distinguished from regions containing ideal adhesive with good contrast. Besides the above mentioned types of defects, kissing bonds of different sizes in the two interfaces of the adhesive have been analyzed. Figure 8(G) illustrates the thermographic results of defects located on the glass side having a size of $20 \mathrm{~mm}$ and 30 mm in diameter. Although irregularities can be recognized, the contrast between ideal and defective adhesive bonds is much lower than in the aforementioned results. This can be explained by the tight mechanical contact between adhesive and glass elements, which has similar thermal properties as accurate regions.

\subsection{Conclusions}

The thermographic testing results of the industrial application have proved that the method of mirror scanning fulfills all requirements such as short inspection cycles, detectability of all quality relevant defects and a high degree of automation. Figure 9 shows the required testing time performances for conventional and mirror scan testing for the aforementioned reference application. The stated times include only the recording time of the infrared camera. As it has been explained in the previous chapters, conventional testing is based on a sequential testing strategy, which leads to inspection times of more than 30 minutes in case of a thick polyurethane bonding. On the contrary, the developed mirror scan is able to parallelize the inspection of up to 20 sections, so that the total testing time depends only on the inspection time for a single section of the 
test object. However, the best performance of thermographic mirror scanning systems compared to conventional testing methods can be achieved if large or widespread areas of interest with slow thermal response behavior need to be inspected.
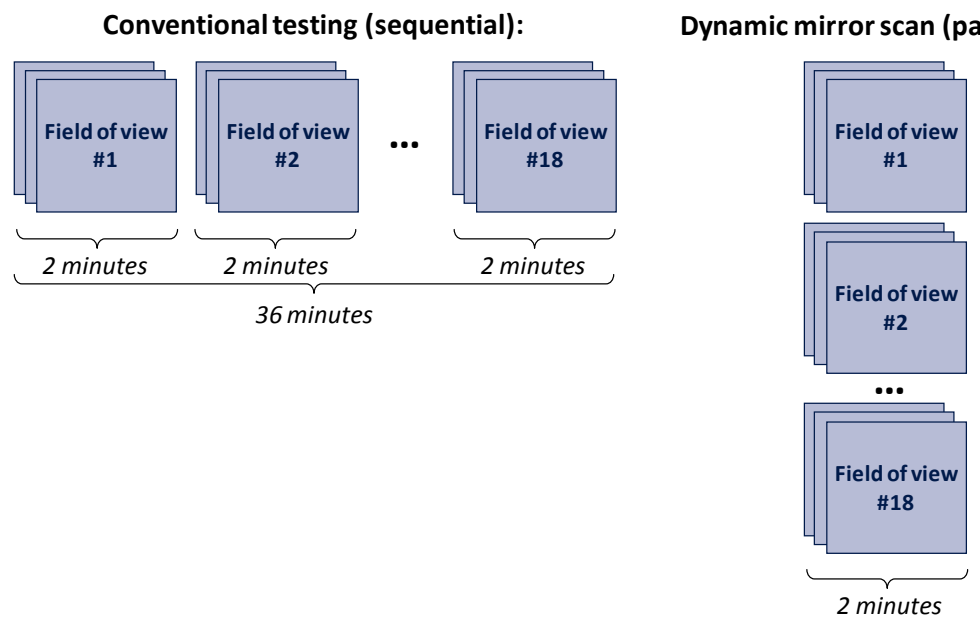

Fig. 9. Reduction of testing time using dynamic mirror scan

\section{Further applications for scanning mirror systems}

Thermography is by far not limited to the inspection of adhesive bonds. This non-destructive testing method has been successfully introduced into the aircraft business as a reference and inspection tool for airplane components. Recent research activities showed the potential of this technology to inspect carbon fiber reinforced plastic (CFRP) components in a fast, contactless and reliable way [11]. Recent airplane production concepts include a great amount of CFRP, for example the body shell of modern airplanes will consist of more than $60 \%$ CFRP. Current inspection methods are based on the conventional sequentially working method as explained in the previous chapters. Thermographic mirror scan might be able to reduce inspection time for a whole airplane of currently one day with high manual effort to less than an hour. Figure 10 shows a theoretical scenario for the application of the described mirror scan system. Further research activities in this field are currently in progress.
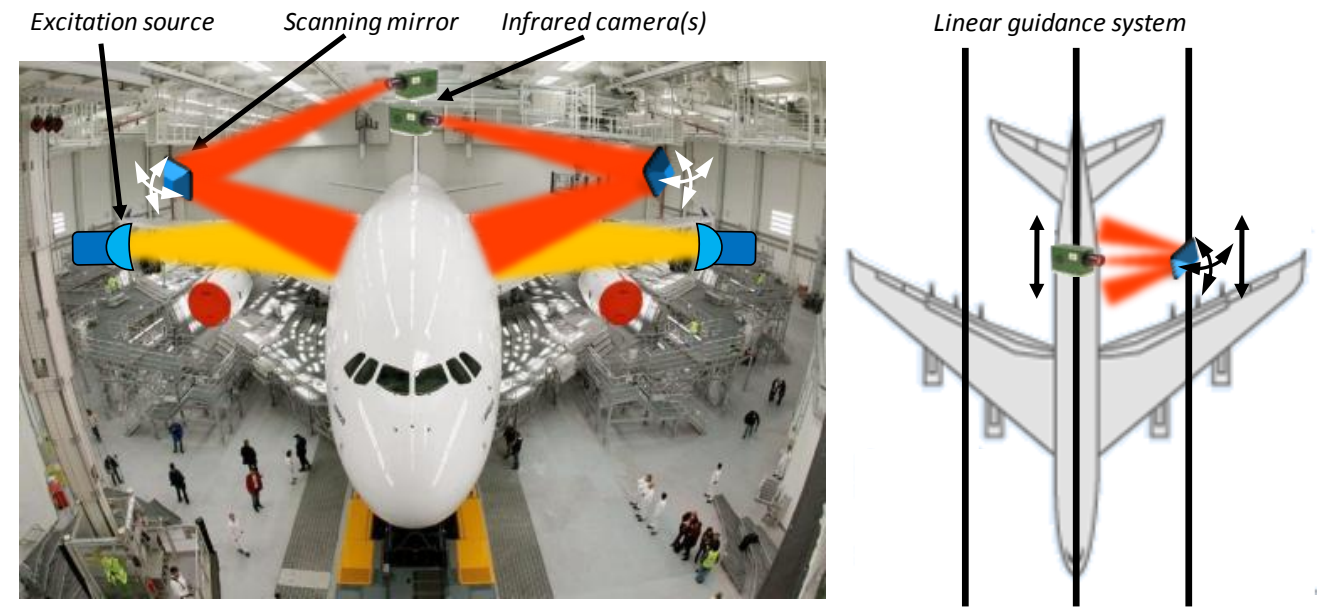

Fig. 10. Scenario-based configuration for the automatic inspection of aircraft structures

\section{Summary}

The enhancement of non-destructive testing methods with respect to a reliable detection of all quality-related defects, short testing periods and a high automation level is of great economical and technical importance for ensuring the operational capability of adhesive bonded parts. Recent research activities proved that active thermography is able to detect various kinds of defects in a broad range of adhesive applications. However, the inspection of large-scale objects using state- 
of-the-art technologies is often too slow for an in-line industrial application, because many adhesive bonds have a slow thermal response behavior. Due to limited camera resolutions large-scale objects cannot be analyzed in a single measurement configuration, so that a separation into smaller sections is necessary. Conventional, sequentially measuring thermography can result in testing times of several minutes when thick layers need to be tested. This paper presented a new concept based on scanning thermography by adjustable mirrors which allows a parallelization of several section measurements. The method has been evaluated by the industrial reference application of a bonded window module which is applied in the automotive industry. Experiments showed the promising potential of scanning thermography as an automated non-destructive testing method for large-scale adhesive bonds. All types of defects relevant to the production process such as interfacial unbonding, interruptions, neckings as well as porosity were detected clearly with high contrast. The inspection time for one test object was reduced by a factor of 18. Future activities will focus on the application of mirror scanning thermography for large CFRP structures, which are widely used in the airplane industry.

\section{REFERENCES}

[1] Berglind, H.; Dillenz, A. (2003): Detection of glue deficiency in laminated wood with pulse thermography. In: J Wood Sci, H. 49, p. 216-220.

[2] Han, X.; Favro, L.D.; Ouyang, Z.; Thomas, R.L. (2001): Thermosonics: Detecting Cracks and Adhesion Defects Using Ultrasonic Excitation and Infrared Imaging. In: The Journal of Adhesion, Jg. 76, H. 2, p. $151-162$.

[3] Hasenberg, D.; Dilger, K.; Boehm, S.: Nondestructive Characterization of Adhesive Joints Using Lock-In Thermography. In: 16th World Conference on Nondestructive Testing. Montreal (Canada), August 30th - September 3rd, 2004.

[4] Zaeh, M. F.; Thiemann, C.; Boehm, S.; Srajbr, C.: Automated non-destructive testing of bonded windows. Joining in Automotive Engineering 2009. Bad Nauheim, $28^{\text {th }} / 29^{\text {th }}$ April 2009.

[5] Zaeh, M. F.; Thiemann, C.; Boehm, S. Srajbr C.; Lammel, C.; Noak, J. (2009): Cost-effective defect detection in bonded glass element modules using active thermography. Non-destructive testing of adhesive bonds. In: Adhesive \& Sealants Extra, H. 10, p. 30-34.

[6] Zettner, J.; Spellenberg, B.; Hierl, T.; Haller, M.; Lenzi, T. (2003): Waermefluss-Prüfung zur Qualitätssicherung von Schweißverbindungen in der Automobilindustrie. In: Deutsche Gesellschaft für Zerstoerungsfreie Prüfung e.V. (Hg.): Thermografie-Kolloquium 2003. Stuttgart, 25.09.2003. Berlin, p. 63-74.

[7] Baumann, J.; Netzelmann, U.; Bilgram, R.; Hierl, T.; Carl, V.; Schubach, H. R.; Zenzinger, G. (2005): Untersuchungen zu aktiven thermographischen Prüfmethoden zur Absicherung von Hochleistungsfertigungsverfahren - Ergebnisse eines BMBF-Projekts. In: Deutsche Gesellschaft für Zerstoerungsfreie Pruefung e.V. (Hg.): DGZfP-Jahrestagung. Rostock, 2.-4. Mai. Berlin.

[8] Wandelt, M. (05/2008): JetCheck: Systemloesung für Inspektionen an Luftfahrzeugen mit aktiver Thermografie. Automation Technology $\mathrm{GmbH}$.

[9] Maldague, X. P. V.; Moore, P. O. (Hg.) (2001): Infrared and thermal testing. 3. ed. Columbus, Ohio: American Society for Nondestructive Testing (Nondestructive testing handbook / Robert C. McMaster, ed. American Society for Nondestructive Testing American Society for Metals, Vol. 3).

[10] Thiemann, C.; Zaeh, M. F.; Srajbr, C.; Boehm, S. (2009): Automatisierte zerstoerungsfreie Pruefung von Klebverbindungen. In: Deutsche Gesellschaft für Zerstoerungsfreie Pruefung e.V. (Hg.): Thermografie-Kolloquium 2009. Stuttgart, 08.-09. Oktober 2009. Berlin.

[11] Cawley, P.: Inspection of Composites - Current Status and Challenges. In: 9th European NDT Conference (ECNDT). Berlin, September 25th-29th, 2006. 